\section{Metallic glasses: the reason why}

\author{
from Robert W. Cahn
}

Metallic glasses in great variety have been made by quenching appropriate liquid alloys at ultrafast rates, $10^{5}-10^{8} \mathrm{~K}$ $\mathrm{s}^{-1}$, into the form of thin ribbons or disks. Most such glasses consist of metal/metalloid combinations but some are also made from metal-metal melts. The degree of (meta)stability of such a glass against crystallisation can be measured in two ways: the critical cooling rate required to inhibit crystallisation during the quench can be estimated, or the tempcrature at which the glass begins to crystallise on reheating can be determined. The former measure, sometimes called 'glass formability', is to be preferred, because it is related to the maximum rate of crystallisation of the glass (located at a temperature well above that of incipient crystallisation); it is the maximum speed at which a crystal can consume the glass that matters, not the temperature at which it happens.

A question which has exercised metallurgists and physicists ever since the first liquid-quenched metallic glass was discovered in 1959 is: what atomic or structural features determine the degree of glass formability? Three alternative models have been proposed. They are not mutually exclusive, but it is nevertheless of considerable interest to know which factor is the crucial one for any particular glass, or even for metallic glasses in general. One model, formulated by Polk in 1972, envisages simply that metallic atoms form a dense random packed array and the smaller metalloid atoms fill the voids in that array and thereby stabilise it. This model cannot be of universal validity, since it cannot account for the formability of the metal-metal glasses. A second model, originated by Chen and Clark in 1973, points to the X-ray evidence for the existence of short-range chemical order between first-nearest neighbour atom pairs in metallic glasses and melts (evidence which has continued to accumulate) and attributes easy glass formability to the enhancement of cohesion in the liquid/glass resulting from strong, chemical bonding between unlike nearest neighbours. The third model, conceived by Nagel and Tauc in 1975, attributes the high resistance of certain glasses to crystallisation to purely electronic factors. This last model, intended as a critique of the other two, seemed to be riding high, but now its claim to universality has been overthrown by a new experimental study (Bauhofer \& Simon Phys. Rev. Lett. 40, 1730; 1978).

Robert W. Cahn is Professor of Materials Science at the University of Sussex.
The free-electron model of S. R. Nagel and J. Tauc (Proc. 2nd Int. Conf. Rapidly Quenched Metals (ed. Grant \& Giessen) 337, MIT Press, 1976; Solid State Comm. 22, 129; 1977) is in effect a modification of Mott and Jones' classical model for the stability of certain crystalline phases such as $\beta$ CuZn. Nagel and Tauc point out that a glass must have a spherical Fermi surface of radius $k_{\mathrm{F}}$ and, although no reciprocal lattice vectors exist, there is an analogous quantity, which is experimentally determinable; this is the structure factor $S(Q)$, a function of reciprocal distance, closely related to the interference function and the pair distribution function. The structure factor of any glass (or liquid) always has a high and narrow first peak, at reciprocal distance $=q_{\mathrm{r}}$, and the main postulate of Nagel and Tauc is that high glass formability is favoured by the condition $2 k_{\mathrm{F}}=q_{\mathrm{P}}$. This is equivalent to the condition that the Fermi energy $E_{\mathrm{F}}$ lies at a minimum of the density of states curve: this in turn implies that the glass reposes in a metastable minimum of electronic energy with respect to changes in composition and therefore in $E_{\mathrm{F}}$. In their 1977 paper they explain, ingeniously, how this criterion can lead naturally to a preference for unlike nearest neighbours without the need to postulate strong interatomic bonds, though the nagging suspicion will not go away that this distinction amounts merely to semantic sleight-of-hand! They also show that a number of actual glasses in the Au-Si, Au-Ge and Co-P systems obey their criterion.

What Bauhofer and Simon have done is to make and examine glasses in two unexpected systems, $\mathrm{Cs}-\mathrm{O}$ and $\mathrm{Rb}-\mathrm{O}$, and show that these glasses do not at all obey the Nagel-Tauc criterion. Though pure alkali metals are stereotypical non glass formers, Cs-O melts can be turned into glasses over the range $15-20$ at. $\% \mathrm{O}$, and $\mathrm{Rb}-\mathrm{O}$ over a narrow range near 13 at. $\% O$ (which does not incidentally coincide with the eutectic minimum at $\sim 9$ at. \% $\mathrm{O}$ ); the $\mathrm{Rb}-\mathrm{O}$ glasses have a small admixture of crystallites. These glasses all crystallise at about $200 \mathrm{~K}$, so that to make them, a sub-zero quenching medium is needed. It turns out that quenching the melt, contained in a $2 \mathrm{~mm}$ capillary, direct into liquid nitrogen is effective, in spite of the fact that the cooling rate cannot exceed $100 \mathrm{~K} \mathrm{~s}^{-1}$.

Bauhofer and Simon also measured the X-ray scattering of their glasses and thus located the first peak of $S(Q)$ in each case. They then adduce evi- dence that oxygen dissolved in liquid caesium removes two electrons per oxygen atom from the conduction band to form an $\mathrm{O}^{2-}$ ion; this also happens in crystalline $\mathrm{Rb}$ and Cs suboxides. They conclude that the Fermi level can be computed on this basis, and when this is done it turns out that, for both glasses, $k_{F}$ coincides accurately with the valley between the first two peaks of the structure factor. Thus, $2 k_{\mathrm{F}}=q_{\mathrm{P}}$ is not obeyed for these very readily formable glasses, and the authors conclude that strong though non-directional chemical bonding in the glasses is the basis of their stability. To form, for instance, crystalline caesium suboxide, large, strongly bonded $\mathrm{Cs}_{11} \mathrm{O}_{3}$ clusters have to form first and this is bound to be slow; presumably similar clusters exist in the glass. The electronic criterion of glass formability, whatever its merits eventually turn out to be, is not universal.

IN July 1878 the editor of Nature, Norman Lockyer, watched the eclipse of the Sun from the station of Separation in Wyoming. "... I give a rough sketch of what I saw of the corona with the naked eye (top), slightly exaggerating the dimensions of the streamers to show the wind-vane appearance, which, to me, was almost perfect, being pointed at one end and bounded by parallel lines at the other; others I may say, however saw a resemblance to a fish's tail. These streamers vanished absolutely in the telescope (bottom), as did the radiation lines in 1871 ; not a shred of them was left.

From Nature 18, 29 August, 457; 1878.
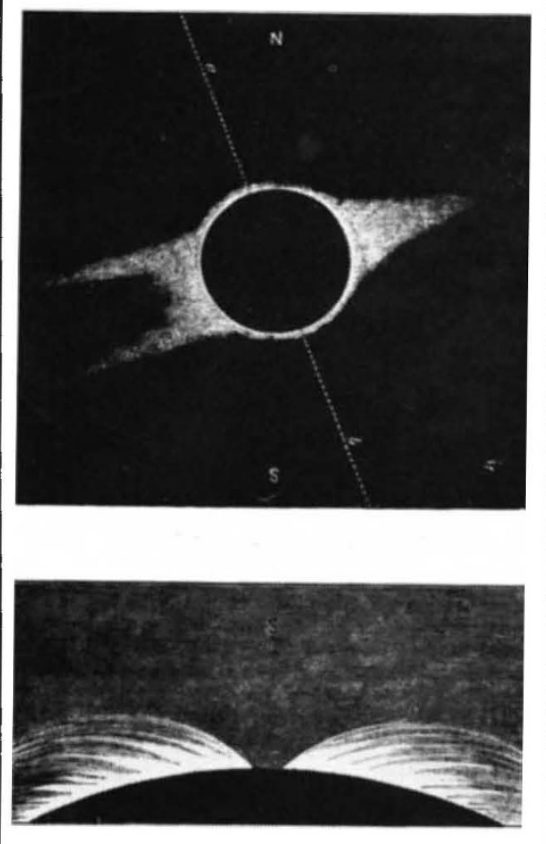